\title{
Evolutionary dynamics analysis of complex network with fusion nodes and overlap edges
}

\author{
YANG Yinghui ${ }^{1,2, *}$, LI Jianhua ${ }^{1}$, SHEN Di ${ }^{1}$, NAN Mingli ${ }^{1,3}$, and CUI Qiong ${ }^{1}$ \\ 1. Information and Navigation College, Air Force Engineering University, Xi' an 710077, China; \\ 2. Unit 95899 of the PLA, Beijing 100076, China; \\ 3. Unit 95881 of the PLA, Beijing 100095, China
}

\begin{abstract}
Multiple complex networks, each with different properties and mutually fused, have the problems that the evolving process is time varying and non-equilibrium, network structures are layered and interlacing, and evolving characteristics are difficult to be measured. On that account, a dynamic evolving model of complex network with fusion nodes and overlap edges (CNFNOEs) is proposed. Firstly, we define some related concepts of CNFNOEs, and analyze the conversion process of fusion relationship and hierarchy relationship. According to the property difference of various nodes and edges, fusion nodes and overlap edges are subsequently split, and then the CNFNOEs is transformed to interlacing layered complex networks (ILCN). Secondly, the node degree saturation and attraction factors are defined. On that basis, the evolution algorithm and the local world evolution model for ILCN are put forward. Moreover, four typical situations of nodes evolution are discussed, and the degree distribution law during evolution is analyzed by means of the mean field method. Numerical simulation results show that nodes unreached degree saturation follow the exponential distribution with an error of no more than $6 \%$; nodes reached degree saturation follow the distribution of their connection capacities with an error of no more than $3 \%$; network weaving coefficients have a positive correlation with the highest probability of new node and initial number of connected edges. The results have verified the feasibility and effectiveness of the model, which provides a new idea and method for exploring CNFNOE's evolving process and law. Also, the model has good application prospects in structure and dynamics research of transportation network, communication network, social contact network, etc.
\end{abstract}

Keywords: complex network with fusion nodes and overlap edges (CNFNOEs), interlacing layered complex networks (ILCN), local world, dynamic evolvement, split, saturation, attraction factor.

DOI: $10.21629 / J S E E .2018 .03 .12$

\footnotetext{
Manuscript received July 29, 2016

*Corresponding author.

This work was supported by the National Natural Science Foundation of China (61573017; 61401499; 61174162).
}

\section{Introduction}

With the increasing of network degree in our society, complex networks have been widely used in the fields such as politics, economy and military $[1,2]$. Currently, complex networks with single property and single edge have drawn much attention [3-5], but complex networks with fusion nodes and overlap edges (CNFNOEs) are rarely concerned. Actually, there are plentiful CNFNOEs in our daily life [6-8] which consist of sub-networks with different characteristics such as traffic network, communication network and human relationship network. These networks are commonly composed of some sub-networks with different characters [7,9], in which each node has one more characters and there are more than one edge between two nodes. An example is the traffic network between cities. The cities can be regarded as nodes and the traffic lines are the edges. A city may have motorway stations, railway stations, and airports simultaneously. Also, there are highroads, railways and lanes between two cities. That is, the traffic network consists of a complex structure with fusion nodes and overlap edges. Compared with complex networks with single property and single edge, the CNFNOEs have more tangled topology and more paths between two nodes $[10,11]$. Dealing with such kind of networks, edges with multiple characters cannot be simply treated as one edge because their characters are quite different (like railway and lane) [12-14].

As for the evolution of complex networks, a new entering node will choose from existing nodes in the classical BA model [3], that is, the "preferential orientation" is prior to the overall information of the network. In general, a new entering node can only acquire information within a range rather than the whole network $[3,15]$, which means the new entering node occupies and uses partial information of the whole network with preferential orientation. For 
example, a computer in the Internet can only connect with another within the same domain. In a real network evolution, the edge selection is bidirectional for a node [16], that is, a new entering node chooses an edge with some conditions, meanwhile the qualified nodes can decide whether it turns out to be a candidate according to autologous factors (such as the cost of new added edge) [17]. Besides, in view of factors like cost, size, environment and so on, nodes in real network hold some capacity [7], when it is reached or approached, the measurement value does not increase anymore and achieves a relative saturation, and the node will not be a candidate. Meanwhile, a large number of living examples suggest that, the edge and increasing ratio of nodes in real networks depend not only on time duration from the entering moment but also on the attraction factor of nodes [8,9]. The higher the attraction factor is, the more edges the node would have. For example, website with better contents will attract a large amount of visitors rapidly, and overnight stars will in no time appeal to plenty of fans even they will easily exceed those who join the entertainment circle earlier.

At present, the achievements about complex network evolution are mainly concentrated in the model construction [1,5,7], algorithm design [3,8], characteristic analysis $[2,4,9]$ and so on. In a recent work [18], under the condition of random attack, the authors investigated the relationship between hierarchical network and the dependence of the intensity on the system based on the scale-free network and Erdôs-Rényi network. Reference [19] proposed a novel evolving bipartite network model based on preferential attachment in the local world, which was generated by node saturation restrictions, not new node selection. In [20], dynamic equations for interdependent "networks of networks" were presented to reproduce the failure cascade for an arbitrary pattern of interdependency. When a fraction of nodes in one network randomly fails, the damage propagates to nodes in networks that are interdependent and a dynamic failure cascade occurs that affects the entire system. Three different kinds of edge patterns, i.e. assortative edge, disassortative edge and random edge, between two interdependent networks could be found in [21]. Taking into account the load, the load redistribution, and the node capacity, the authors study the robustness of two interdependent networks. The authors in [22] discussed evolutionary dynamics of distinct networks (layers) of interdependent networks, and explored an update rule in which revision of strategies was a biased imitation process. In these networks, imitation and interaction between individuals of opposite layers are established through interlinks. The existing results deepen the complex network research on the evolutionary dynamics. However, previous studies mainly have failed to distinguish a variety of properties of the nodes and edges of the evolutionary modeling, and only a little attention has been focused on evolving characteristics and the law of CNFNOEs.

In this paper, for the dynamic evolving issue of CNFNOEs, some concepts concerning are firstly defined, and then CNFNOE is transformed into interlacing layered complex networks (ILCN) according to character differences, splitting fusion nodes and coincidence edges. Secondly, the definition of saturation values and attraction factors of nodes are proposed respectively. Subsequently, the construction algorithm of the dynamic evolving model and the ILCN partially dynamic evolving model are given. Furthermore, the value distribution regularities are calculated theoretically. Finally, the validity and effectiveness of the proposed model are verified through simulations.

\section{CNFNOE model transformations}

\subsection{Concepts}

Definition 1 Fusion nodes are comprehensive ones with more than two characteristics (like transportations, transmission rate, time delay and medium). They are more likely the rendezvous points of multi-characters edges which are denoted as $\boldsymbol{V}^{*}=\bigcup_{i=1}^{m} V_{i}^{*}$, where $m$ is the number of fusion nodes and each fusion node possesses $P\left(V_{i}^{*}\right)$ kinds of characters.

Definition 2 Overlap edges refer to edges with two more characters (the partition method of characters keeps consistent with that of fusion nodes), which are denoted as $\boldsymbol{E}^{*}=\bigcup_{i=1}^{n} E_{i}^{*}$, where $n$ is the number of overlap edges. Each overlap edge $E_{i}^{*}$ possesses $P\left(E_{i}^{*}\right)$ kinds of characters and it gives $P\left(E_{i}^{*}\right)=P\left(V_{i}^{*}\right)$ in general in a same CNFNOE.

Definition 3 CNFNOEs refer to compound networks consisting of sub-networks with absolutely or partially same nodes and close but not equal edges. It can be denoted as $C_{M}\left(\boldsymbol{V}_{M}^{*}, \boldsymbol{V}_{M}, \boldsymbol{E}_{M}^{*}, \boldsymbol{E}_{M}, \boldsymbol{T}_{M}\right)$, where $\boldsymbol{V}_{M}^{*}$ is a set of fusion nodes, $\boldsymbol{V}_{M}$ is a set of non-fusion nodes, $\boldsymbol{E}_{M}^{*}$ and $\boldsymbol{E}_{M}$ keep the same, respectively. $\boldsymbol{T}_{M}=\left(t_{i j}\right)_{N_{M} \times N_{M}}$ is an adjoining matrix, where $N_{M}=\left|\boldsymbol{V}_{M}^{*} \cup \boldsymbol{V}_{M}\right|$, and $t_{i j} \in\{0,1, \ldots, n\}$ denotes edges with different characters.

Definition 4 ILCN consists of two or more interlacing layered sub-networks with partial nodes connecting and edges possessing close but not equal characters [23]. It can be denoted as $C_{C}\left(\boldsymbol{V}_{C}, \boldsymbol{E}_{C}, \boldsymbol{T}_{C}, \boldsymbol{\varphi}_{C}\right)$, where $\boldsymbol{V}_{C}$ is 
a set of nodes, $\boldsymbol{E}_{C}$ is a set of edges, $\boldsymbol{T}_{C}=\left(t_{i j}\right)_{N_{C} \times N_{C}}$ is an adjoining matrix where $N_{C}=\left|\boldsymbol{V}_{C}\right|, \boldsymbol{\varphi}_{C}$ is a matrix of network interlacing factor which is used to describe the intimacy level of interlacing relationship between two networks and the calculation method was described in [24].

\subsection{Transformation of fusion relationship and hierarchy relationship}

United relationship and hierarchy relationship are all to describe the structure of networks and are laid particular emphasis on different aspects [25]. Fusion relationship pays more attention to the overall properties and makes contribution to analysis on comprehensive properties of nodes (edges), feasible paths of networks and connection relationship of networks. Fusion relationship can effectively support rank setting of nodes, information itinerary planning and so on. Hierarchy relationship can describe the separated topology structure of different sub-networks and makes contribution to layered property analysis of networks. Hierarchy relationship can effectively support task allocation, functional partitioning, and decentralized management and so on. Fusion relationship and hierarchy relationship can also make mutual transformation with the change of united extent of sub-networks.

Take two sub-networks for example, as is shown in Fig. 1. When the number of fusion nodes is $\left|\boldsymbol{V}_{M}^{*}\right|=1$, there is no hierarchy relationship in the network topology but connecting heterogeneous networks. When $\left|\boldsymbol{V}_{M}^{*}\right|=3$, as is shown in Fig. 1(b) and Fig. 1(d), there are general characters of double layered networks while it still meets the definition of CNFNOE described in the paper. Thus, layered networks can be seen as an advantaged stage in the process of fusion networks evolution.

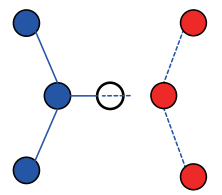

(a) Fusion relationship when $\left|\boldsymbol{V}_{M}^{*}\right|=1$

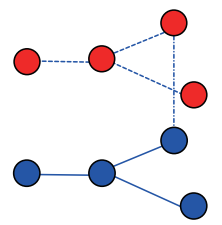

(c) Hierarchy relationship when $\left|\boldsymbol{V}_{M}^{*}\right|=1$

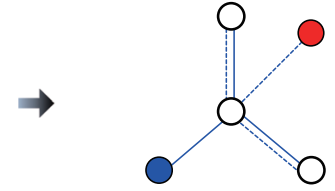

(b) Fusion relationship when $\left|\boldsymbol{V}_{M}^{*}\right|=3$

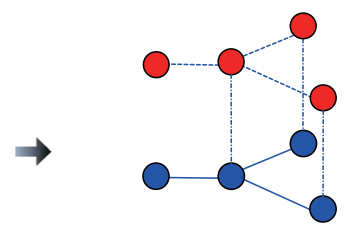

(d) Hierarchy relationship when $\left|\boldsymbol{V}_{M}^{*}\right|=3$
Fig. 1 Sketch map of fusion relationship and hierarchy relationship evolution

\subsection{Split operation}

On account of the multiple properties of fusion nodes, some inconvenience is brought into the property attribution during the evolution processing of CNFNOE. Hence, the properties of new nodes are hardly to be distinguished in the optimization process of new nodes. Meanwhile, the external forms of different edges are presented as one edge because of the existence of overlap edges. Some difficulties and inaccuracies are brought into the statistic of the degree of existed nodes. Whereas, in ILCN, each node and each edge are relatively independent, so fusion and coincident conditions do not exist, which is beneficial to researching the evolution regulation of sub-networks with different properties [26]. Hence, in this paper, CNFNOE is converted as ILCN by the split operation of fusion nodes and overlap edges based on property difference. The split operations are classified as fusion node split, overlap edges split and whole network split based on different research objects.

\subsubsection{Fusion node split}

Each fusion node is split into several different nodes with single property based on the property difference of nodes. The total connected community structure is formed by connections of the new edges, whose properties are generally different from those of the overlap edges between the nodes after split. In this way, sub-networks of different properties are cross level connected, and an interweaving network of the hierarchical structure is formed. Supposing the fusion node $V_{i}^{*}$ with property $P\left(V_{i}^{*}\right)$, the split operation is defined as

$$
V_{i}^{*} \stackrel{f_{V^{*}}}{\longrightarrow} C_{i}=\bigcup_{k=1}^{P\left(V_{i}^{*}\right)} v_{i, k} \cup\left(\frac{P\left(V_{i}^{*}\right) \cdot\left[P\left(V_{i}^{*}\right)-1\right]}{\bigcup_{t=1}^{2}} e_{t}\right)
$$

where $f_{V^{*}}$ is the split function of fusion nodes, $C_{i}$ is the total connected network after the split of $V_{i}^{*}, v_{i, k}$ are the nodes of single property after split which amount to $P\left(V_{i}^{*}\right)$, and $e_{t}$ is the new internal edge during the split process, which amounts to $\frac{P\left(V_{i}^{*}\right) \cdot\left[P\left(V_{i}^{*}\right)-1\right]}{2}$.

CNFNOE consisting of the nodes and the edges of three properties is taken for instance, and the process of fusion nodes split is shown in Fig. 2. $V_{w 1}^{*}$ and $V_{w 2}^{*}$ are fusion nodes. $V_{w 1}^{*}$ can be split as $V_{3}^{a}, V_{3}^{b}$ and $V_{3}^{c}$, and $V_{w 2}^{*}$ can be split as $V_{4}^{a}, V_{4}^{b}$ and $V_{4}^{c}$. These two groups of node sets are totally internal connected, which bridge the gap between the correlation of the hierarchical networks $V_{4}^{c}, V_{4}^{c}$ and $V_{4}^{c}$. For a convenient description, the nodes with the same property but different quantity are all regarded as the same node. 


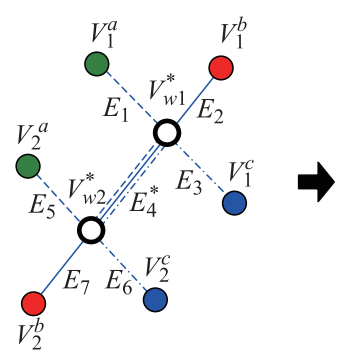

(a) CNFNOE

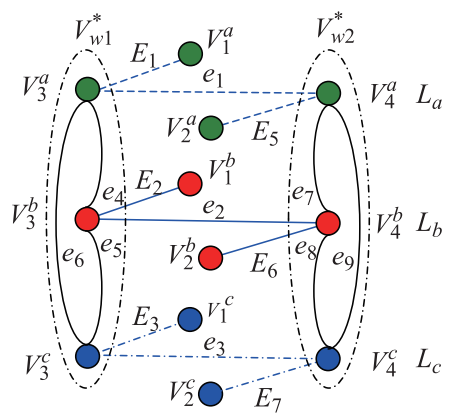

(b) ILCN
Fig. 2 Schematic diagram of the convert process of CNFNOE and ILCN

\subsubsection{Overlap edge split}

Based on the differences of edges, the overlap edges are split into edges with single property, and as the internal edges of the sub-network, they connect the fusion nodes after split [27]. Supposing the overlap edge $E_{i}^{*}$ has the property $P\left(E_{i}^{*}\right)$, the split process is defined as

$$
E_{i}^{*} \stackrel{f_{E^{*}}}{\longrightarrow} \bigcup_{k=1}^{P\left(E_{i}^{*}\right)} e_{k}
$$

where $f_{E^{*}}$ is the split function of overlap edges, $e_{k}$ denotes the edge with single property after split, which amounts to $P\left(E_{i}^{*}\right)$. Particularly, when $P\left(E_{i}^{*}\right)=1$, the network is a complex network of single property, which can be split. However, when split, the sub-network may not connect even there are isolate nodes.

In Fig. 2, the overlap edge $E_{4}^{*}$ exists between the nodes $V_{w 1}^{*}$ and $V_{w 2}^{*}$, which possess three kinds of properties presented as different line forms. $E_{4}^{+}$can be split as three edges $e_{1}, e_{2}$ and $e_{3}$, which consists of the internal edges of $L_{a}, L_{b}, L_{c}$.

\subsubsection{Whole network split}

Based on the split of fusion nodes and overlap edges, the whole network can be split as several mono-property networks, some of which may not be connected network. The operation of whole network split is defined as

$$
\begin{aligned}
& C_{M} \stackrel{f_{C^{*}}}{\longrightarrow} C_{C}=\boldsymbol{V}_{M} \cup\left(\bigcup_{i=1}^{\left|\boldsymbol{V}_{M}^{*}\right| P\left(V_{i}^{*}\right)} \bigcup_{k=1} v_{i, k}\right) \cup \\
& \boldsymbol{E}_{M} \cup\left(\bigcup_{k=1}^{P\left(E_{i}^{*}\right)} e_{k}\right) \cup\left(\frac{P\left(V_{i}^{*}\right) \cdot\left[P\left(V_{i}^{*}\right)-1\right]}{\bigcup_{t=1}^{2}} e_{t}\right)
\end{aligned}
$$

where $f_{C^{*}}$ is the split function of the whole network, which can be presented as the combining function of $f_{V^{*}}$ and $f_{E^{*}}$, denoted by $f_{C^{*}}=F\left(f_{V^{*}}, f_{E^{*}}\right)$. The node set of network $C_{C}$ is $\boldsymbol{V}_{C}=\boldsymbol{V}_{M} \cup\left(\bigcup_{i=1}^{\left|\boldsymbol{V}_{M}^{*}\right|} \bigcup_{k=1}^{P\left(V_{i}^{*}\right)} v_{i, k}\right)$, and the edge set is $\boldsymbol{E}_{C}=\boldsymbol{E}_{M} \cup\left(\bigcup_{k=1}^{P\left(E_{i}^{*}\right)} e_{k}\right) \cup$ $\left(\frac{P\left(V_{i}^{*}\right) \cdot\left[P\left(V_{i}^{*}\right)-1\right]}{\bigcup_{t=1}^{2}} e_{t}\right)$

In Fig. 2(b), $V_{1}^{a}-V_{4}^{a}$ constitutes the sub-network $L_{a}$, $V_{1}^{b}-V_{4}^{b}$ constitutes the sub-network $L_{b}$, and $V_{1}^{c}-V_{4}^{c}$ constitutes the sub-network $L_{c} . L_{a}, L_{b}$ and $L_{c}$ correlate with each other by nodes $V_{a 3}, V_{b 3}, V_{c 3}, V_{a 4}, V_{b 4}$ and $V_{c 4}$, and thus ILCN is formed.

\section{ILCN dynamic evolution model}

Different from the BA scale-free network, most nodes in realistic network have the ability of variant edge growth influenced by management system, cost and volume. These nodes have a certain connection capacity (upper the limit of the connection amount), the nodes which can be connected with are limited and edges cannot increase without limits. For better balance and survivability, new nodes will be preferred to connect with the nodes from the node set of low degree saturation $[21,28]$. Meanwhile, the existed nodes in the network have their own attraction factors, which will influence the preferred process and results of new nodes. Hence, in this paper, the degree saturation is considered as the judgment criteria of whether the existed nodes join into the local world and become the candidate nodes. The attraction factor is determined as the main basis of connection priority of the new nodes. Based on these criteria, the dynamic evolution model of ILCN is constructed.

\subsection{Node degree saturation and attraction factor}

\subsubsection{Node degree saturation}

Definition 6 Node degree saturation is a piecewise function between the node connection capacity and the degree. The difference of the node degree in the evolution network can be decreased by the limited degree saturation, which makes a relative uniform distribution of the generated edges, and improves the balance of the whole network. The degree saturation function of node $V_{i}$ at the evolution time $t$ is presented as

$$
B_{i}(t)=\left\{\begin{array}{l}
\Omega_{i}, \quad k_{i}(t)=0 \\
\Theta\left(\Omega_{i}, k_{i}(t)\right), \quad k_{i}(t) \in\left(0, k^{*}\right) \\
\Omega^{*}, \quad k_{i}(t)=k^{*}
\end{array}\right.
$$

where $\Omega_{i}$ is the connection capacity of node $V_{i}$, which can make step change by upgrade, dilatation and remoulding. $k_{i}(t)$ is the degree of node $V_{i}, k^{*}$ is the degree thre- 
shold of the node, and $\Omega^{*}$ is the relative steady-state value. $\Theta\left(\Omega_{\iota}^{P}, k_{i}^{P}\right)$ is the degree saturation function of the node, which takes direct proportion to the change tendency of $\Omega_{i}$, and to $k_{i}(t)$ inversely. If $\Omega_{i}$ is relatively invariant, $B_{i}(t)$ decreases as $k_{i}(t)$ increases at the beginning. When $k_{i}(t)$ reaches the threshold $k^{*}, B_{i}(t)$ will almost not change and equals $\Omega^{*}$, though $k_{i}(t)$ increases consistently.

The node degree saturation is determined as the judgment criteria of whether enlarge the edges of the existed nodes in the network. The dynamic local-world of the new nodes is generated passively. The new nodes in different node sets can only be preferred selected in the unsaturated node sets of the corresponding kinds. The node set is the local-world of new nodes. It is not determined by the new nodes but generates automatically based on the bearing capacity of the existed nodes after judgment. To state conveniently, in this paper, $B_{i}(t)=\Omega_{i}-k_{i}(t)$ denotes the degree saturation function, where $k_{i}(t) \in\left[0, \Omega_{i}\right]$. If $B_{i}(t)=0$ or $k_{i}(t)=\Omega_{i}$, it denotes that the node is saturated and does not accept new edges, which will not connect with the new nodes. If $B_{i}(t)>0$ or $k_{i}(t)<\Omega_{i}$, it denotes that the node can build edges with other nodes and become the connection object of the local-world.

\subsubsection{Node attraction factor}

Definition 7 The node attraction factor refers to the number of nodes connected in a unit time. In the real case, the node attraction factor is used to represent as the complex attracting variable of the existing node to a new node, and it generally is proportional to the adding number of edges in the unit time sides. As for node $V_{i}$, attraction factor $\Upsilon_{i}$ can be expressed as

$$
\Upsilon_{i}=\frac{\Delta E}{\Delta t}
$$

where $\Delta t$ is the time interval, and $\Delta E$ is the number of edges obtained in the $\Delta t$.

Different from the BA model, the presence of the attraction factor owns a great impact on the preferred selective probability of new nodes; even nodes have only a few connections with the high attraction factor [29]. Although these nodes appear in the network for a little time, they have a high connection rate and build a lot of connections in a short time, which makes them reach the saturation.

\subsection{Evolution model construction algorithm}

Set the connection capacity matrix of the node is $\boldsymbol{\Omega}=$ $\left[\Omega_{i}\right]_{m_{0} \times 1}$ at the initial time, where $m_{0}$ is the number of nodes. Each node has new probabilities, and it connects with the same property nodes preferentially. When the number of the unsaturated node which has the same property with new nodes is smaller than the initial edge num- ber of the new node, the new node will select other property nodes which are unsaturated according to the node degree and the attraction factor. When the properties of new nodes and selected nodes are determined, the property of edges is determined [30]. Therefore, the property and probability of edges are not considered in the processing of evolution. The evolutionary model construction algorithm is as follows:

Step 1 Growth: Starting on fewer nodes $m_{0}$ and edges $e_{0}$, add a single property node in each step in the processing of evolution, the new probability of node with property $S$ is $p_{S}\left(0 \leqslant p_{S} \leqslant 1\right)$, and it connects to $m\left(m \leqslant m_{0}\right)$ existing nodes with the same type.

Step 2 Determine the local-world: According to the degree of the same type nodes in the network at the evolution time $t$, calculate the degree saturation of each node, and the local-world of the same type nodes will generate naturally.

Step 3 Connection: The connection probability between new nodes with property $S$ and the node $V_{i}^{S}$ in the same type local-world depends on the node degree $k_{i}^{S}$ and the attraction factor $\Upsilon_{i}^{S}$, i.e.

$$
\prod_{\text {local }}\left(V_{i}^{S}\right)=p_{S} \cdot \frac{M}{t p_{S}+m_{0}^{S}} \cdot \frac{k_{i}^{S}+\Upsilon_{i}^{S}}{\sum_{\text {local }}\left(k_{i}^{S}+\Upsilon_{i}^{S}\right)}
$$

where $m_{0}^{S}$ is the number of nodes with property $S$ in the initial network, $M$ is the number of nodes in the localworld of the same type nodes, and $\sum_{\text {local }}\left(k_{i}^{S}+\Upsilon_{i}^{S}\right)$ is the sum of the same type nodes degree and the attraction factor in the local-world.

In order to describe the evolution law in detail and predict the degree distribution, assume $k$ is successive, then the increased rate of node $V_{i}^{S}$ degree is

$$
\frac{\partial k_{i}^{S}}{\partial t}=\frac{M m p_{S}}{t p_{S}+m_{0}^{S}} \cdot \frac{k_{i}^{S}+\Upsilon_{i}^{S}}{\sum_{\text {local }}\left(k_{i}^{S}+\Upsilon_{i}^{S}\right)} .
$$

After $t$ evolution steps, the algorithm can produce a network with $t+m_{0}$ nodes (where the number of property $S$ nodes is $\left.t p_{S}+m_{0}^{S}\right)$ and $m t+e_{0}$ edges.

\subsection{Discussion of the nodes evolution in different situations}

Affected by the nodes' degree saturation and the attraction factor, the nodes in ILCN evolution will be divided into four different situations, as shown in Fig. 3. The number of the white hole in the node represents the inherent connection capacity. 

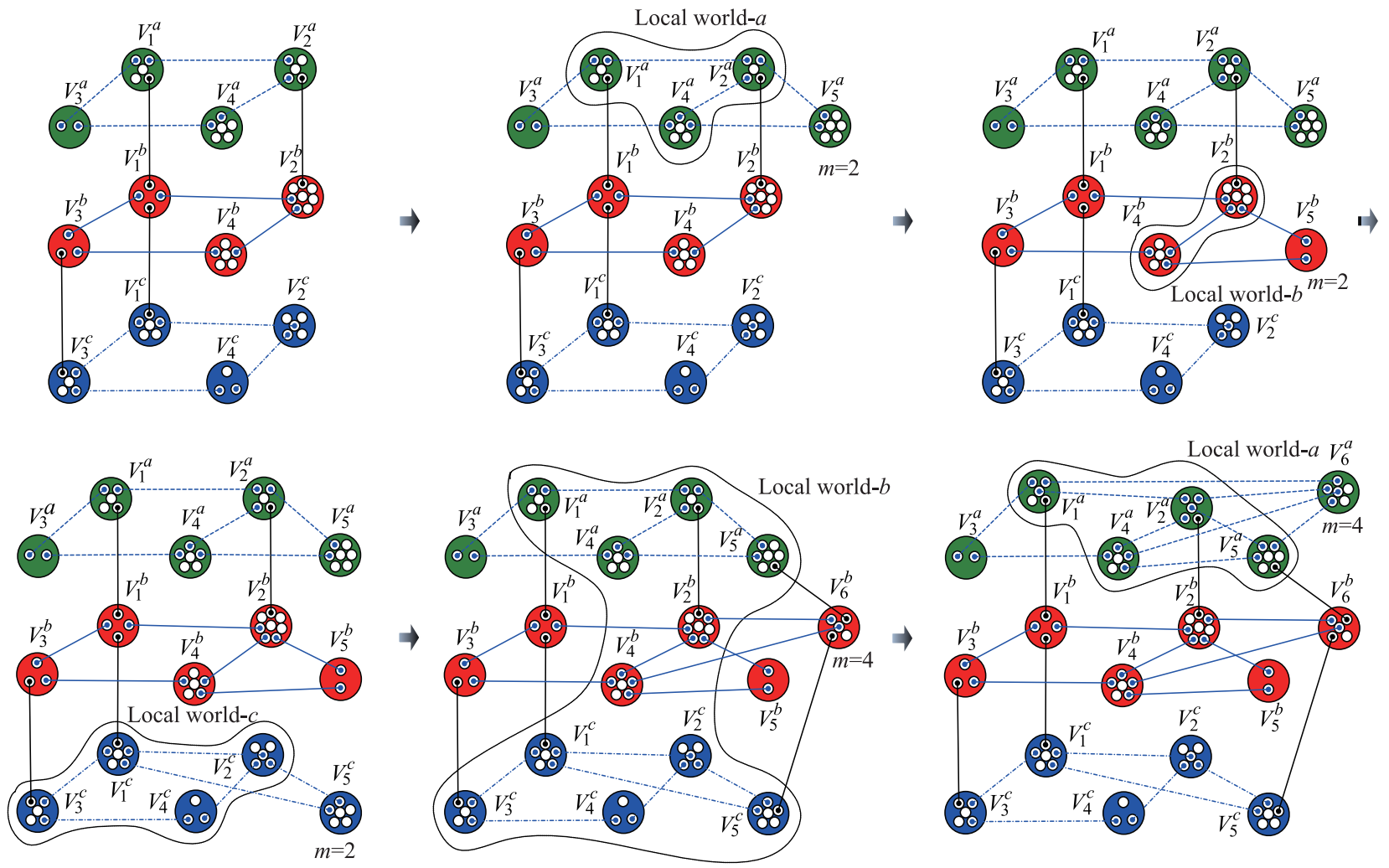

Fig. 3 Flow chart of ILCN evolution

(i) When $B_{i}$ and $\Upsilon_{i}$ are large, such nodes have great advantages in connecting with the same kind of new nodes, and they will get more multilateral in few evolutionary steps, which will gradually evolve into the distributed nodes, such as the node $V_{2}^{a}$. In addition, they may become distributed nodes before the old nodes. For example, although the node $V_{5}^{a}$ joins the network for not a long time, it will get connections with other new nodes quickly owing to its large attraction factor and become the distributed node before the node $V_{3}^{a}$.

(ii) When $B_{i}$ is large and $\Upsilon_{i}$ is small, such nodes do not have the advantage in connecting with the same kind of new node, which is equivalent to an old weak node. Although it owns a larger saturation, its attraction factor is too small and in the evolution processing there will be no change. As shown in Fig. 3, although nodes $V_{1}^{a}$ and $V_{2}^{a}$ are the initial nodes in the network, the attractive factor of node $V_{1}^{a}$ is smaller and the increase of its degree is not obvious.

(iii) When $B_{i}$ is small, $\Upsilon_{i}$ is large, such nodes will connect with new nodes quickly in evolution processing, however, there is no connection priority compared to the case (i) owing to saturation capacity. In addition, when the node degree reaches the relatively saturated, the nodes remain stable, and there is no connection with the time how long the nodes are in the network, like the node $V_{1}^{c}$.

(iv) When $B_{i}$ and $\Upsilon_{i}$ are small, the connection probability of such nodes is the lowest in these four cases. The number of edges increases very slowly, and they will gradually evolve into the marginal nodes, like the node $V_{4}^{c}$.

\subsection{Node degree distribution analysis}

The node degree distributions are divided into two kinds of extreme situations, which are restricted by the node connection capacity:

(i) The connection capacity $\Omega_{i}$ of the node is large, and each node degree cannot reach saturated at the end of evolution.

With the mean field method, node degree distribution can be analyzed in this situation [31]. There is only a priority connection pattern in ILCN evolution. Assume

$$
\begin{gathered}
\sum_{\text {local }}\left(k_{i}^{S}+\Upsilon_{i}^{S}\right)=\left\langle k_{i}^{S}+\Upsilon_{i}^{S}\right\rangle \cdot M \\
\left\langle k_{i}^{S}+\Upsilon_{i}^{S}\right\rangle=\left\langle k_{i}^{S}\right\rangle+\left\langle\Upsilon_{i}^{S}\right\rangle .
\end{gathered}
$$

After the evolution time $t$, the average degree $\left\langle k_{i}^{S}\right\rangle$ is

$$
\left\langle k_{i}^{S}\right\rangle=\frac{2 m t p_{S}+e_{0}^{S}}{t p_{S}+m_{0}^{S}}
$$

where $e_{0}^{S}$ is the number of edges with property $S$ in the initial network. 
Average attraction factor $\left\langle\Upsilon_{i}^{S}\right\rangle$ is

$$
\left\langle\Upsilon_{i}^{S}\right\rangle=\frac{\Delta E}{\Delta t}=\frac{2 m t p_{S}}{t}=2 m p_{S}
$$

Substitute (9) to (11) into (8), and we can obtain

$$
\sum_{\text {local }}\left(k_{i}^{S}+\Upsilon_{i}^{S}\right)=\left(\frac{2 m t p_{S}+e_{0}^{S}}{t p_{S}+m_{0}^{S}}+2 m p_{S}\right) \cdot M \text {. }
$$

Combine (7) and (12), and we can get

$$
\frac{\partial k_{i}^{S}}{\partial t}=\frac{m p_{S}\left(k_{i}^{S}+\Upsilon_{i}^{S}\right)}{2 m p_{S}\left(t+t p_{S}+m_{0}^{S}\right)+e_{0}^{S}}
$$

Considering the initial conditions, that means when the node $V_{i}^{S}$ joins the network at time $t^{*}, k_{i}^{S}\left(t^{*}\right)=m p_{S}$, $\Upsilon_{i}^{S}\left(t^{*}\right)=0$. After solving (13), we could have

$$
\begin{gathered}
k_{i}^{S}=\frac{m p_{S}}{2\left(1+p_{S}\right)-\ln \left[2 m p_{S}\left(t+t p_{S}+m_{0}^{S}\right)+e_{0}^{S}\right]} . \\
\ln \frac{e^{2\left(1+p_{S}\right)}}{2 m p_{S}\left(t^{*}+t^{*} p_{S}+m_{0}^{S}\right)+e_{0}^{S}} .
\end{gathered}
$$

When $k_{i}^{S} \geqslant 0$ and the function of $t$ is monotonically increasing, the probability of $k_{i}^{S}(t)$ can be written as

$$
\begin{gathered}
P\left(k_{i}^{S}<k\right)=p\left\{\frac{m p_{S}}{2\left(1+p_{S}\right)-\ln \left[2 m p_{S}\left(t+t p_{S}+m_{0}^{S}\right)+e_{0}^{S}\right]} \cdot \ln \frac{e^{2\left(1+p_{S}\right)}}{2 m p_{S}\left(t^{*}+t^{*} p_{S}+m_{0}^{S}\right)+e_{0}^{S}}<k\right\}= \\
p\left\{t^{*}>\frac{e}{2 m p_{S}\left(1+p_{S}\right)} \cdot\left[\frac{2}{e} m p_{S}\left(t+t p_{S}+m_{0}^{S}\right)+\frac{1}{e} e_{0}^{S}\right]^{\frac{k}{m p_{S}}}-\frac{m_{0}^{S}}{1+p_{S}}-\frac{e_{0}^{S}}{2 m p_{S}\left(1+p_{S}\right)}\right\}= \\
1-p\left\{t^{*} \leqslant \frac{e}{2 m p_{S}\left(1+p_{S}\right)} \cdot\left[\frac{2}{e} m p_{S}\left(t+t p_{S}+m_{0}^{S}\right)+\frac{1}{e} e_{0}^{S}\right]^{\frac{k}{m p_{S}}}-\frac{m_{0}^{S}}{1+p_{S}}-\frac{e_{0}^{S}}{2 m p_{S}\left(1+p_{S}\right)}\right\} .
\end{gathered}
$$

Assume that the new nodes join the network in the same time interval, and the time $t$ will be subject to the uniform distribution, i.e.

$$
P\left(t^{*}\right)=\frac{1}{t p_{S}+m_{0}^{S}} .
$$

Thus

$$
\begin{gathered}
P\left(k_{i}^{S}<k\right)= \\
1-\frac{1}{m_{0}^{S}+t p_{S}} \cdot\left\{\left[\frac{2}{e} m p_{S}\left(t+t p_{S}+m_{0}^{S}\right)+\frac{1}{e} e_{0}^{S}\right]^{\frac{k}{m p_{S}}} .\right. \\
\left.\frac{e}{2 m\left(1+p_{S}\right)}-\frac{p_{S} m_{0}^{S}}{1+p_{S}}-\frac{e_{0}^{S}}{2 m\left(1+p_{S}\right)}\right\} .
\end{gathered}
$$

The probability density of $k^{S}(t)$ is

$$
\begin{gathered}
P\left(k^{S}\right)=\frac{\partial P\left[k_{i}^{S}<k\right]}{\partial k}= \\
\frac{e-\ln \left[2 m p_{S}\left(t+t p_{S}+m_{0}^{S}\right)+e_{0}^{S}\right]^{e}}{2 m^{2} p_{S}\left(1+p_{S}\right)\left(t p_{S}+m_{0}^{S}\right)} . \\
{\left[\frac{2}{e} m p_{S}\left(t+t p_{S}+m_{0}^{S}\right)+\frac{1}{e} e_{0}^{S}\right]^{\frac{k}{m p_{S}}} .}
\end{gathered}
$$

When $t \rightarrow \infty$, the node degree distribution is

$$
P\left(k^{S}\right) \sim \frac{e^{2 p_{S}\left(1+p_{S}\right)}}{4 m^{2} p_{S}^{2}} \cdot e^{-\frac{2\left(1+p_{S}\right)}{m} \cdot k} .
$$

Obviously, the node degree distribution of the ILCN model follows the exponential distribution, and the index will be in -4 to 0 by adjusting the parameters $m$ and $p_{S}$. (ii) The connection capacity $\Omega_{i}$ of the node is small, and each node degree reaches saturated at the end of evolution.

In this situation, the node degree is equal to the connection capacity at the end of evolution, so the node degree distribution is consistent with the connection capacity distribution, and it may be subject to a fixed distribution or a random distribution. Actually, each node connection capacity is determined in consideration of cost, benefit, demands and the external environment conditions [32].

Generally, ILCN model evolution interposes between the two kinds of extreme conditions, and the distribution of node degree usually takes the form of mixed distribution, which means that unsaturated node degree distribution follows an exponential distribution and saturated node degree distribution is consistent with the connection capacity distribution.

\section{Simulations}

We use Matlab to simulate the evolution processing of the ILCN model, and the evolution step is 100 steps. We assume that the connection capacity distribution of the node obeys the normal distribution from 1 to $10 . \Omega \sim$ $N(4.8,2.7)$ and the factors such as upgrade and expansion of the nodes in the evolution are not taken into consideration. At the initial time, the number of nodes $m_{0}=6$ in CNFNOE, and the properties of nodes are divided into $a$ (green), $b$ (red), $c$ (blue) and $p_{a}, p_{b}, p_{c}$ are the probabilities of new nodes of these three categories $a, b, c$ respectively. The fusion node $V_{2}^{*}-V_{5}^{*}$ is white, the number of 
edges $e_{0}=5$ (coincident edges are regarded as an edge). In addition, the edges are divided into $a$ (dotted lines), $b$ (solid line) and $c$ (dash dot line), and the edges between $V_{2}^{*}$ and $V_{3}^{*}$, and $V_{4}^{*}$ and $V_{5}^{*}$ are coincident edges. The initial CNFNOE model is shown in Fig. 4(a).

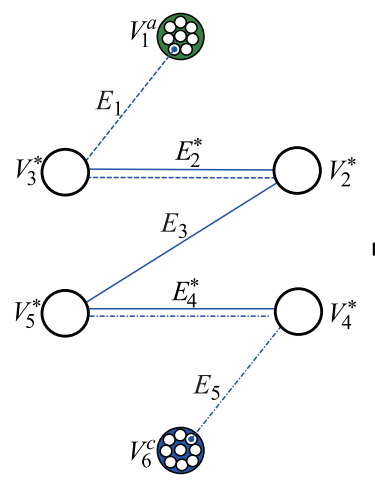

(a) CNFNOE

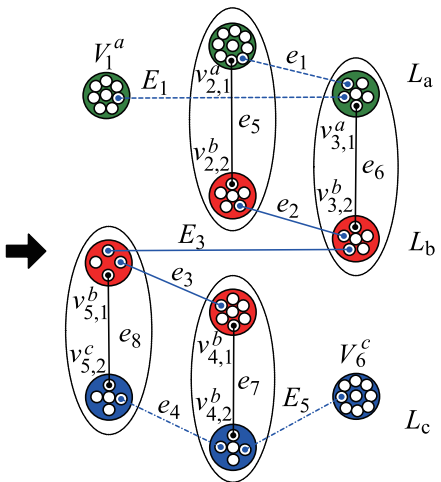

(b) ILCN
Fig. 4 Initial CNFNOE model and the transformed ILCN model

\subsection{CNFNOE model transformation}

According to property difference, split the fusion nodes as $V_{2}^{*} \rightarrow\left\{v_{2,1}^{a}, v_{2,2}^{b} \mid e_{5}\right\}, V_{3}^{*} \rightarrow\left\{v_{3,1}^{a}, v_{3,2}^{b} \mid e_{6}\right\}, V_{4}^{*} \rightarrow$ $\left\{v_{4,1}^{b}, v_{4,2}^{c} \mid e_{7}\right\}, V_{5}^{*} \rightarrow\left\{v_{5,1}^{b}, v_{5,2}^{c} \mid e_{8}\right\}$, where $e_{5}-e_{8}$ denote the additional edges after splitting the fusion nodes. And split the overlap edges as $E_{2}^{*} \rightarrow\left\{e_{1}, e_{2}\right\}, E_{4}^{*} \rightarrow\left\{e_{3}, e_{4}\right\}$. The nodes $V_{1}^{a}, V_{6}^{c}$ and the edges $E_{1}, E_{3}, E_{5}$ are not affected by the split operations and have remained the same. After splitting, the nodes $V_{1}^{a}, v_{2,1}^{a}, v_{3,1}^{a}$ and the edges $E_{1}$, $e_{1}$ form the subnet $L_{a}$, the nodes $v_{2,2}^{b}, v_{3,2}^{b}, v_{4,1}^{b}, v_{5,1}^{b}$ and the edges $E_{3}, e_{2}, e_{3}$ form the subnet $L_{b}$, the nodes $v_{4,2}^{c}$, $v_{5,2}^{c}, V_{6}^{c}$ and the edges $E_{5}, e_{4}$ form the subnet $L_{c} . L_{a}, L_{b}$ and $L_{c}$ obey the hierarchical structure distribution, $L_{a}$ and $L_{b}, L_{b}$ and $L_{c}$ are intertwined with each other. The ILCN model is as shown in Fig. 4(b).

\subsection{Node degree distribution in probability combination of $p_{a}, p_{b}$ and $p_{c}$ when $m$ is fixed}

When $m=1$, the degree distribution of various nodes in the network versus the probability combination of $p_{a}, p_{b}$ and $p_{c}$ are shown in Fig. 5. From this figure, for any curve of single distribution, probability values are generally decreasing with the node degree $k$ increasing, and the probability values present the typical exponential distribution when $k$ reaches a certain value. The reason for this is that $m$ is a small value. After adding the new nodes into the network, the influence on the existing node degree is slow. At the end of the evolution, the majority of the nodes in the network are still in the unsaturated state, and only a small number of nodes with smaller capacity reach the degree of saturation. When $p_{a}=0.2, p_{b}=0.3, p_{c}=0.5$ and $m=1$, for the $b$ set of nodes, only three nodes in 24 nodes reach the degree of saturation, which accounts for $12.5 \%$ of the total. According to the simulation method, the node degree value is determined to obey the exponential distribution, and the index $\lambda$ is 2.773 . Taking $p_{b}$ and $m$ into (18), theoretical calculation of the exponential value is $2\left(1+p_{b}\right) / m=2.6$. The simulation results are in agreement with the mean field method and the error is only about $6 \%$, which can verify the correctness of the theoretical calculation of the model. The reason for the error is that the evolutionary step in the numerical simulation is limited to only 100 steps and the theoretical prediction is the result of the calculation when $t$ is infinite. As a result, the simulation results and the theoretical calculation will be closer when the step size and the network size are larger.

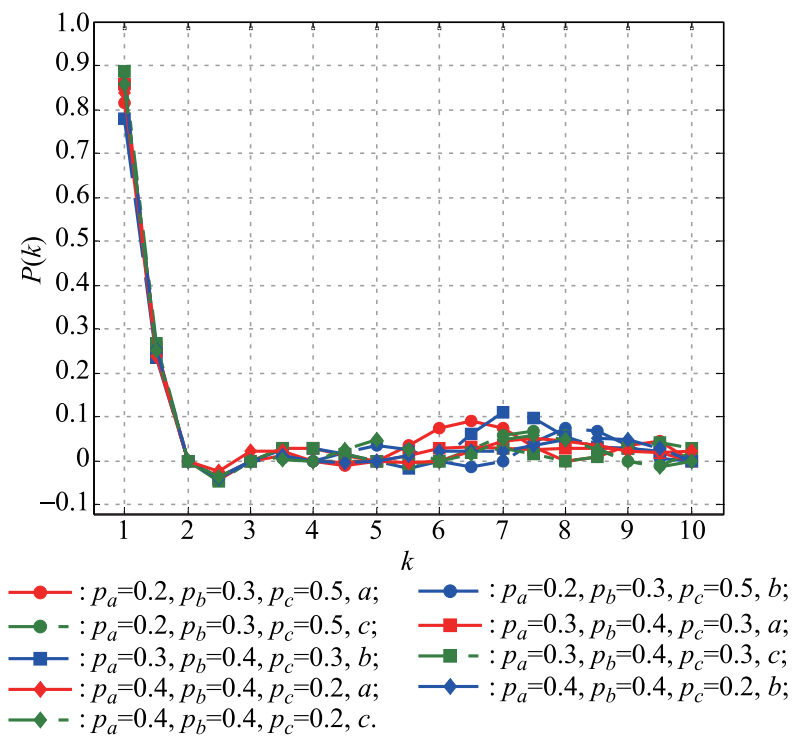

Fig. 5 Degree distribution of the nodes in the network versus $p_{a}$, $p_{b}$ and $p_{c}$ when $m=1$

According to the comprehensive comparison of the nine curves, taking $m=1$ and the $b$ set of nodes as example, the exponential $\lambda$ is 2.773 by fitting when $p_{a}=0.2, p_{b}=0.3$, $p_{c}=0.5$, and is 2.876 when $p_{a}=0.3, p_{b}=0.4, p_{c}=0.5$. The exponential $\lambda$ results of two probability combinations are basically consistent and the error is only about $3.6 \%$. When $m$ is the same, although the probability combination of $p_{a}, p_{b}$ and $p_{c}$ is different, the probability of only a few degrees of nodes in the network produces fluctuation, and the degree distribution curves of the nodes have little change, which are still subject to exponential distribution overall. The simulation results show that the probability of the new node is not the key factor to affect the distribution of nodes degree in the proposed model. 


\subsection{Node degree distribution versus $m$ when the probability combination of} $p_{a}, p_{b}$ and $p_{c}$ is fixed

When $p_{a}=0.2, p_{b}=0.3$ and $p_{c}=0.5$, the degree distributions of various nodes in the network versus the $m$ from 1 to 3 are as shown in Fig. 6. From this figure, the distribution curves of the $a$ set of nodes, $b$ set of nodes and $c$ set of nodes obey the exponential distribution when $m=1$. However, with the gradual increasing of $m$, the probabilities of the degree from 1 to 3 in the curves are greatly reduced, the probabilities of the degree from 4 to 7 in the curves have obvious promotion, the probabilities of the degree from 8 to 10 in the curves are almost constant, and only partial intervals of the curve obey the exponential distribution. The reason for this is as follows: the initial number of edges of the new nodes joining the network is more than 2 when $m \geqslant 2$, the number of the low-degree nodes in the network decreases ceaselessly caused by the rapid increase of node degree in the network in the subsequent evolution, and the greater the $m$ is, the faster the node degree increases and the smaller the probability of occurrence is. Because the total length of the evolution is significantly larger than the connection capacity of each node, at the end of the evolution, most of the nodes are gradually close to the connection capacity, reach the saturation state and stay in the vicinity of the connection capacity. By fitting the distribution of these nodes reached degree saturation, $\mu=4.9$ and $\sigma^{2}=3.0$ can be obtained, where $P(k) \sim N(4.9,3.0)$ is in agreement with the simulation setting $\Omega \sim N(4.8,2.7)$. The mathematical expectation error is about $2 \%$ and the variance error is about $3 \%$. By fitting the distribution of these $b$ nodes unreached degree saturation with $m=2, \lambda=1.43$ can be obtained.

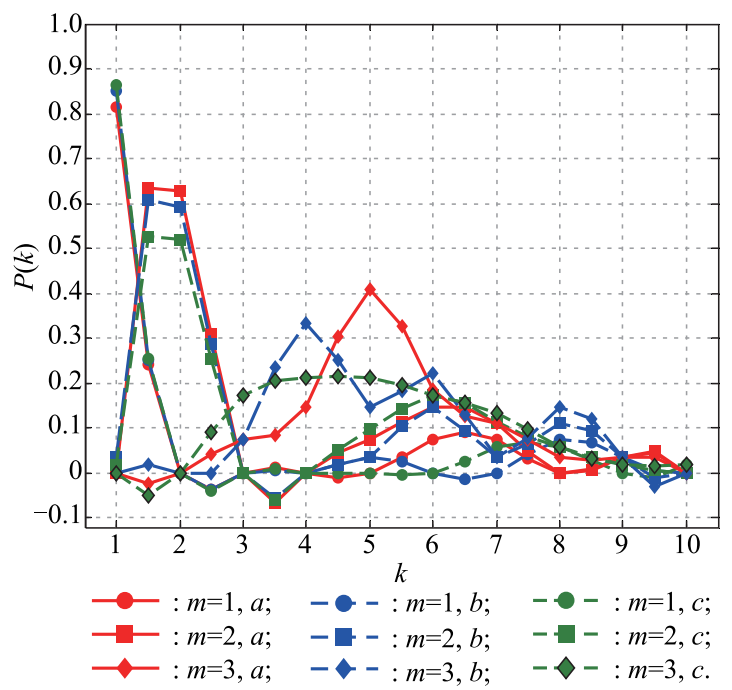

Fig. 6 Degree distribution of the nodes in the network versus the increasing $m$ when $p_{a}=0.2, p_{b}=0.3$ and $p_{c}=0.5$
Taking $p_{b}$ and $m$ into (18), theoretical calculation of the exponential value is $2\left(1+p_{b}\right) / m=1.3$. The simulation results are in agreement with the theoretical calculation value and the error is only about $5 \%$.

In addition, the probabilities of $a, b$ and $c$ nodes whose degrees are above 5 are respectively $37.1 \%, 37.2 \%$ and $46.2 \%$ when $m=1,18.5 \%, 11.1 \%$ and $13.5 \%$ when $m=2$, and $77.8 \%, 59.3 \%$ and $61.5 \%$ when $m=3$. It can be seen as follows: when the new node with more edges (i.e. the larger $m$ ) establishes a connection with the existing nodes, degrees of the nodes in the network generally are relatively large and the ratio of high-degree nodes is high, which is in agreement with the real network.

\subsection{Trends of network weaving coefficients $\varphi_{c}$ versus $m$ and the probability combination of $p_{a}, p_{b}$ and $p_{c}$}

When two sets of values are taken for $m$ and the probability combination of $p_{a}, p_{b}$ and $p_{c}$, the variation of the network weaving coefficients of ILCN with the step size is shown in Fig. 7. From this figure, the network weaving coefficient between any two layer networks presents the step type oscillation with the evolution of the propulsion, and eventually tends to be relatively stable. When the probability combination is fixed, the greater the value of $m$ is, the greater the network weaving coefficient is. For example, when $p_{a}=0.3, p_{b}=0.4$ and $p_{c}=0.3$, the network weaving coefficient of $L_{a}-L_{b}$ with $m=2$ is significantly higher than the coefficient with $m=1$. This is because the larger $m$ requires more connections between new nodes and the existed nodes in the network. When the number of the similar unsaturated nodes is smaller than $m$, the new nodes will select to connect with the other categories of unsaturated nodes (in other network layer), which increases the number of the weaving nodes between different networks to promote node weaving degree of the network.

When $m$ is fixed, the network weaving coefficient of the new nodes with higher probability is larger. For example, when $m=2, p_{a}=0.2, p_{b}=0.3$ and $p_{c}=0.5$, the network weaving coefficient of $L_{b}-L_{c}$ is significantly higher than the coefficient with $p_{a}=0.3, p_{b}=0.4$ and $p_{c}=0.3$. In this case, the probability of $c$ nodes is higher and the new $c$ set of nodes is more than $a$ set of nodes and $b$ set of nodes. Due to the preferential attachment mechanism, the node degrees in $L_{c}$ are generally larger and increase faster, even some nodes reach degree saturation in advance. To meet the requests of the number of connections, new nodes will bring the other types of nodes which are unsaturated into the local-world, and preferentially establish the connections to increase the number of weaving 
edges between the other types of nodes, which makes the weaving relationships of the edges in the network denser and more complex.

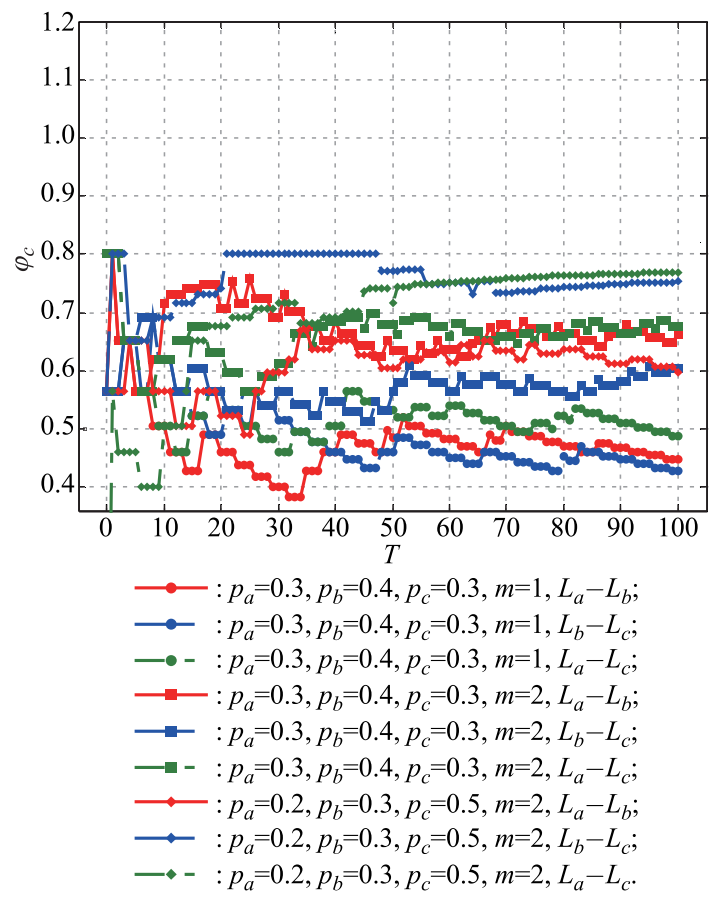

Fig. 7 Trends of network weaving coefficients $\varphi_{c}$ versus $\boldsymbol{m}$ and the probability combination of $p_{a}, p_{b}$ and $p_{c}$

\section{Conclusions}

In this paper, to explore the evolving characteristics of CNFNOE, we transform the CNFNOE model to the ILCN model by split operation, and build a dynamic evolving model based on the corporate effect of nodes' degree saturation and attraction factor. The node degree distribution and the variation of the network weaving coefficients are analyzed by simulations. The proposed model provides a new way to study the evolutionary dynamics of CNFNOE, and main conclusions are as follows:

(i) Under the corporate effect of nodes' degree saturation and attraction factor, all nodes in the network obey the mixed distribution law. The nodes which are unsaturated obey the exponential distribution law, and the nodes reaching degree saturation obey their connection capacities' distribution law. The parameter value of the mixed distribution is closely related to the value of $m$, but is not strongly related to the type probability of the new nodes.

(ii) The network weaving coefficients have a positive correlation with the highest probability of new nodes and the initial number of connected edges. Along with gradual advance of the evolutionary step, the coefficients surge by step and finally tend to be relatively stable.
In order to learn more about the evolution characteristics and the law of the CNFNOE model, we will focus on the topics such as the problems of the node extinction, addition of the multiple nodes to the network at the same time, and changes of the edge value in the heterogeneous network in the future work.

\section{References}

[1] CASTET J F, SALEH J H. Interdependent multi-layer networks: modeling and survivability analysis with applications to space-based networks. PLoS One, 2013, 8(4): e60402.

[2] WANG Z, SZOLNOKI A, PERC M. Self-organization towards optimally interdependent networks by means of coevolution. New Journal of Physics, 2014, 16(3): 033041.

[3] BARABASI A L, ALBERT R. Emergence of scaling in random networks. Science, 1999, 286(5439): 509-512.

[4] WATTS D J, STROGATZ S H. Collective dynamics of smallworld networks. Nature, 1998, 393(6684): 440-442.

[5] AXELROD R, HAMILTON W D. The evolution of cooperation. Science, 1981, 211(4489): 1390-1396.

[6] OHTSUKI H, HAUERT C, LIEBERMAN E, et al. A simple rule for the evolution of cooperation on graphs and social networks. Nature, 2006(441): 502-505.

[7] KURANT M, THIRAN P. Layered complex networks. Physical Review Letters, 2006, 96 (13): 138701.

[8] AOKI T, YAWATA K, AOYAGI T. Self-organization of complex networks as a dynamical system. Physical Review E, 2015, 91(1): 012908.

[9] GOMEZ-GARDENES J, GRACIA-LAZARO C, FLORA L $\mathrm{M}$, et al. Evolutionary dynamics on interdependent populations. Physical Review E, 2012, 86(2): 056113.

[10] NICOSIA V, BIANCONI G, LATORA V, et al. Growing multiplex networks. Physical Review Letters, 2013, 111(5): 058701.

[11] GAO J, BULDYREV S V, STANLEY H E, et al. Networks formed from interdependent networks. Nature Physics, 2012, 8(1): $40-48$.

[12] BULDYREV S V, SHERE N W, CWILICH G A. Interdependent networks with identical degrees of mutually dependent nodes. Physical Review E, 2011, 83(1): 016112.

[13] GOMEZ-GARDENES J, REINARES I, ARENAS A, et al. Evolution of cooperation in multiplex networks. Scientific Reports, 2012(2): 620 .

[14] ZOU S R, ZHOU T, LIU A F. Topological relation of layered complex network. Physics Letters A, 2010, 374(43): 4406 4410.

[15] ROCA C P, CUESTA J A, SANCHEZ A. Effect of spatial structure on the evolution of cooperation. Physical Review E, 2009, 80(4): 046106.

[16] OHTSUKI H, NOWAK M A, PACHECO J M. Breaking the symmetry between interaction and replacement in evolutionary dynamics on graphs. Physical Review Letters, 2007, 98(10): 108106.

[17] NOWAK M A. Five rules for the evolution of cooperation. Science, 2006, 314(5805): 1560-1563.

[18] JIANG J, LI W, CAI X. The effect of interdependence on the percolation of interdependent networks. Physica A, 2014, 410(12): $573-581$.

[19] TIAN L X, HE Y H, HUANG Y. A novel local-world-like evolving bipartite network model. Acta Physica Sinica, 2012, 61(22): 228903.

[20] VALDEZ L D, MACRI P A, STANLEY H E, et al. Triple point in correlated interdependent networks. Physical Review 
E, 2013, 88(5): 050803.

[21] WANG J W, JIANG C, QIAN J F. Robustness of interdependent networks with different link patterns against cascading failures. Physica A, 2014, 393(1): 535-541.

[22] SANTOS M D, DOROGOVTSEV S N, MENDES J F F. Biased imitation in coupled evolutionary games in interdependent networks. Scientific Reports, 2014, 4(4): 4436.

[23] PARSHANI R, ROZENBLAT C, IETRI D, et al. Intersimilarity between coupled networks. Europhysics Letters. 2010, 92(6): 68002.

[24] SHEN D, LI J H, ZHANG Q, et al. Interlacing layered complex networks. Acta Physica Sinica, 2014, 63(19): 190201.

[25] GU C G, ZOU S R, XU L. Onset of cooperation between layered networks. Physical Review E, 2011, 84(2): 026101.

[26] DOROGOVTSEV S N, MENDES J F. Evolution of networks. Advances in Physics, 2003, 51(4): 1079-1187.

[27] SHAO S, HUANG X Q, STANLEY H E, et al. Robustness of a partially interdependent network formed of clustered networks. Physical Review E, 2014, 89(3): 032812.

[28] ESQUIVEL-GO'MEZ J, STEVENS-NAVARRO E, PINEDARICO U, et al. A growth model for directed complex networks with power-law shape in the out-degree distribution. Scientific Reports, 2015(5): 7670.

[29] TAO S H, ZHAO H Y, PING Y, et al. Attractive factors based scale-free networks evolving model. Complex Systems and Complexity Science, 2008, 5(2): 88-92.

[30] WANG B K, PEI Z H, WANG L. Evolutionary dynamics of cooperation on interdependent networks with the prisoner's dilemma and snowdrift game. Europhysics Letters, 2014(107): 58006.

[31] JALILI M, SICHANI O A, YU X H. Optimal pinning controllability of complex networks: dependence on network structure. Physical Review E, 2015, 91(1): 012803.

[32] WANG Z, SZOLNOKI A, PERC M. Evolution of public cooperation on interdependent networks: the impact of biased utility functions. Europhysics Letters, 2012(97): 48001.

\section{Biographies}

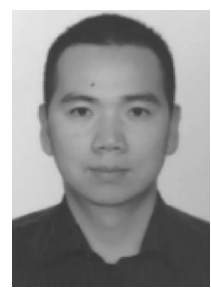

YANG Yinghui was born in 1988. He received his M.S. degree in military command from Air Force Engineering University in 2012 and $\mathrm{Ph} . \mathrm{D}$. degree in 2016 in the same university and profession. He is currently working at a military research institution and his research interests are information flowing and operations modeling.

E-mail: yangyinghui.good@163.com

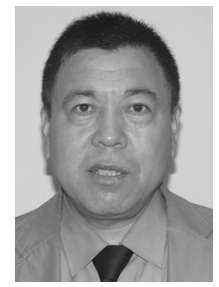

LI Jianhua was born in 1965. He received his M.S. degree in tactical communication from Communication Command Academy in 1994. He received his B.S. degree in military communication from Air Force Engineering University in 2005. Since 1991, he joined Air Force Early Warning Academy, where he is currently a professor and doctor postgraduate tutor. He has taken charge of over ten research subjects and has won eight prizes of army science and technology progress. He has published seven textbooks, and more than 90 papers on international and domestic academic journals. His current research interests are military information system design and construction.

E-mail: KGDLJH@163.com

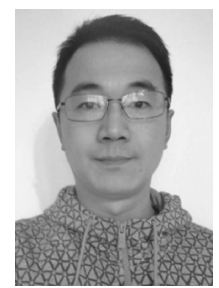

SHEN Di was born in 1986. He received his B.E., M.E. and Ph.D. degrees from Air Force Engineering University in 2008, 2010, 2014 respectively. His research interests include complex network modeling and simulation.

E-mail: hanshanyueyin@126.com

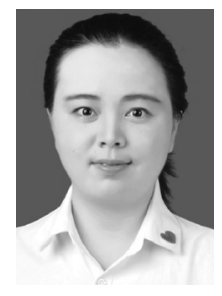

NAN Mingli was born in 1987. She is an M.E. candidate in Air Force Engineering University and received her B.E. degree in 2012. Her research interests include network optimization and simulation. E-mail: nanmingli_good@163.com

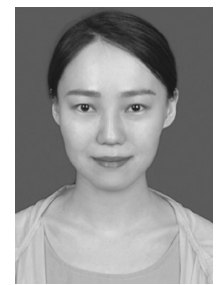

CUI Qiong was born in 1990. She is a Ph.D. candidate in Air Force Engineering University (AFEU) and received her B.E. and M.E. degrees from AFEU in 2011 and 2013 respectively. Her research interests include complex adaptive modeling.

E-mail: flying1990@163.com 